this model. Tape stripping increased the number of tuft cells in the small intestine and their expression of Il25 mRNA. Mice lacking tuft cellspecific expression of IL-25 did not have increased numbers of intestinal mast cells after tape stripping.

Group 2 innate lymphoid cells (ILC2s) are known targets for IL-33 and IL-25, and experiments with mice lacking ILC2s showed that they are required for the mast cell response to tape stripping. Tape stripping increased the number of ILC2s in the small intestine and increased their expression of Il4, Il5 and Ill3 mRNAs. This response was abolished in mice lacking keratinocyte-specific IL-33 expression or tuft cell-specific IL-25 expression, as well as in mice lacking ILC2 expression of the receptors for IL-33 or for IL-25. Thus, IL-33 and IL-25 have direct, nonredundant effects on ILC2s.

Previous work has shown that tuft cells and ILC2s are involved in a feedforward loop during intestinal helminth infection. Similarly, here, the authors showed that keratinocyte-derived IL-33

a Gram-negative mucus-dwelling component of the murine intestinal microbiota. Further experiments showed that Mucispirillum was detectable in $\mathrm{Nod2}^{-1-}$ and $\mathrm{Cy} \mathrm{bb}^{-1-}$ Tac-fostered mice but only showed marked accumulation in the Nod2 $2^{-1-}$ $\mathrm{Cybb}^{-1-}$ Tac-fostered mice. Notably, although Mucispirillum was increased in the Tac-fostered $\mathrm{Nod}^{-1-} \mathrm{Cybb}^{-1-}$ mice before the onset of colitis, after the development of colitis higher levels of Mucispirillum were detected in inflamed intestinal regions.

The authors found that pre-colitic Tac-fostered $\mathrm{Nod}^{-1-} \mathrm{Cybb}^{-/-}$mice have fewer neutrophils in the intestinal lumen and lamina propria than control Tac-fostered wild-type mice, but their neutrophils contained higher levels of Mucispirillum. Further experiments suggested that impaired recruitment and killing activity of neutrophils in the Nod2 $2^{--} \mathrm{Cybb}^{-/-}$mice leads to the accumulation of Mucispirillum and onset of colitis in these animals. The authors also found that maternal $\lg G$ and $\lg A$ antibodies specific for Mucispirillum protect against colitis development in Tac-fostered Nod2 ${ }^{-1-} \mathrm{Cybb}^{-/-}$mice, stimulates ILC2s to produce IL-4 and IL- 13 that stimulate IL-25 production by tuft cells, which further promotes ILC2 activation. IL-4 and IL-13 also signal directly to mast cells, such that in tape-stripped mice with ILC2-specific deficiency of IL-4 and IL-13 or with mast cellspecific deficiency of IL- 4 receptor and IL-13 receptor, there is a decreased oral anaphylaxis response.

The authors suggest that this pathway connecting mechanical skin injury to intestinal responses evolved to protect the gut from helminth infections that breach the skin barrier. Its relevance to atopic responses in humans was shown by the expanded mast cell populations observed in duodenal biopsies from patients with atopic dermatitis compared with controls, independent of the presence of food allergy or of the serum concentration of $\operatorname{IgE}$.

Kirsty Minton

ORIGINAL ARTICLE Leyva-Castillo, J.-M. et al. Mechanical skin injury promotes food anaphylaxis by driving intestinal mast cell expansion. Immunity https://doi.org/10.1016/j.immuni.2019.03.023 (2019)

explaining why disease occurs shortly after weaning.

Finally, to definitively show Mucispirillum induces colitis in Nod2 ${ }^{-1-} \mathrm{Cybb}^{-1-}$ mice, Jax-fostered groups of mice lacking the bacterium were pretreated with vancomycin and orally gavaged with Mucispirillum. Wild-type, Nod2-/- $\mathrm{Cybb}^{-/-}$and Nod2 ${ }^{-/-}$ $\mathrm{Cybb}^{-1-}$ mice showed comparable levels of colonization by Mucispirillum -

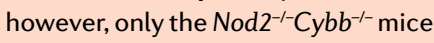
developed colitis-like disease.

Mucispirillum is unlikely to contribute to Crohn's disease in humans, but related bacteria - such as Proteobacteria - have been found to be increased in patients with Crohn's disease. The protective role for antibodies described in this study suggests that the identification of potentially colitogenic bacterial species in humans could enable the development of therapeutic vaccines for patients with Crohn's disease. Yvonne Bordon

ORIGINAL ARTICLE Caruso, R. et al. A specific gene-microbe interaction drives the development of Crohn's disease-like colitis in mice. Sci. Immunol. 4, eaaw4341 (2019)

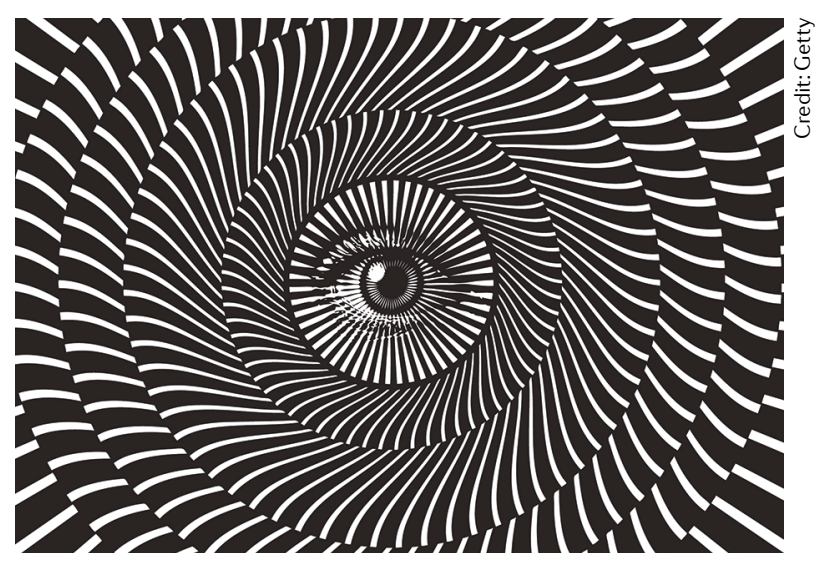

INNATE IMMUNITY

\section{The enigma of circular RNA}

Circular RNAs (circRNAs), which are generated through back-splicing of pre-mRNAs, are stable and widely expressed, but their biological role has remained a mystery. Writing in Cell, Liu et al. now show that circRNAs bind to and thereby 'shield' protein kinase $\mathrm{R}$ (PKR), a pattern recognition receptor with antiviral function that is normally activated by double-stranded RNA (dsRNA).

Previous studies had implicated circRNAs in innate immune regulation. For example, it was found that the biogenesis of nascent circRNAs is reduced upon viral infection and that enforced expression of circRNAs via plasmids can facilitate viral infection of human cells. Moreover, patients with a genetic defect leading to the accumulation of intron-lariat-derived RNA circles are more susceptible to viral infections.

The authors now demonstrate that stimulation of HeLa cells with poly(l:C) or infection with the RNA virus encephalomyocarditis virus induces $R$ Nase L activation, which leads to circRNA degradation and the release of PKR inhibition. The key role of RNaseL in degrading circRNA was confirmed in RNaseL-knockout and RNaseL-knockdown cells and by using a synthetic RNaseL activator.

PKR is known to undergo auto-phosphorylation in response to activation by long dsRNAs ( $>33 \mathrm{bp}$ ), but this activation is blocked by short dsRNAs (16-33 bp). The authors show that circRNAs, but not their linear cognate RNAs, inhibit dsRNA-induced phosphorylation of PKR. Structural analysis of the circRNAs revealed that PKR suppression was independent of their sequence but required the presence of dsRNA regions, which were typically $16-26$ bp in length.

Interestingly, the authors also found that peripheral blood mononuclear cells derived from patients with systemic lupus erythematosus (SLE) contained less circRNAs, had spontaneous RNaseL activation and showed enhanced PKR phosphorylation compared with controls. Overexpression of circRNAs, but not their linear counterparts, attenuated aberrant PKR activation in these cells.

These studies demonstrate that circRNAs can modulate innate immunity to viruses and reveal an unexpected connection between circRNAs and the autoimmune disease SLE.

Alexandra Flemming

ORIGINAL ARTICLE Liu, C.-X. et al. Structure and degradation of circular RNAs regulate PKR activation in innate immunity. Cell https://doi.org/10.1016/j.cell.2019. 03.046 (2019) 